International Journal of Modern Physics A

(C) World Scientific Publishing Company

\title{
SPECTRUM AND DECAYS OF HADRONIC ATOMS
}

\author{
JULIA SCHWEIZER* \\ Institute for Theoretical Physics, University of Bern, Sidlerstr. 5 \\ CH-3012 Bern, Switzerland
}

\begin{abstract}
We describe the spectra and decays of $\pi^{+} \pi^{-}$and $\pi^{ \pm} K^{\mp}$ atoms within a non-relativistic effective field theory. The evaluations of the energy shifts and widths are performed at next-to-leading order in isospin symmetry breaking. The prediction for the lifetime of the $\pi^{ \pm} K^{\mp}$ atom in its ground-state yields $\tau=(3.7 \pm 0.4) \cdot 10^{-15} \mathrm{~s}$.
\end{abstract}

Keywords: Non relativistic effective Lagrangians; Chiral perturbation theory; Isospin symmetry breaking.

\section{Introduction}

Hadronic atoms are highly non-relativistic loosely bound systems of hadrons mainly formed by the Coulomb interaction. The study of these hadronic bound states has seen growing interest during recent years, because they provide important information on the behavior of QCD at low-energy. The DIRAC collaboration 1 at CERN measures the lifetime of the $\pi^{+} \pi^{-}$atom, and plans to determine the $\pi \pi$ scattering lengths difference $\left|a_{0}^{0}-a_{0}^{2}\right|$ at $5 \%$ accuracy. Particularly exiting is the fact that in this manner the current understanding of the $\mathrm{SU}(2) \times \mathrm{SU}(2)$ chiral symmetry breaking in QCD can be verified experimentally. Another experiment on hadronic atoms is presently performed at PSI. The pionic hydrogen collaboration ${ }^{[2]}$ plans to measure the strong energy shift and decay width of the $\pi^{-} p$ atom in its ground-state at the $1 \%$ level. This high precision measurement aims to determine the S-wave $\pi N$ scattering lengths. Finally, the DEAR collaboration 3 at the DA $\Phi$ NE facility measures the strong energy shift and width of the ground-state in kaonic hydrogen. Here, the goal is to extract the $K N$ S-wave scattering lengths.

In order to extract the scattering lengths from such precision measurements, the theoretical expressions for the spectrum and decays must be known to a precision that matches the experimental accuracy. Nearly fifty years ago, Deser et al. $\frac{4}{4}$ derived the leading order formulae for the decay width and the energy shift in pionic hydrogen. Similar relations exist for $\pi^{+} \pi^{-}$and $\pi^{-} K^{+}$atoms $\frac{56}{5}$, which decay predominantly through the strong transitions $\pi^{+} \pi^{-} \rightarrow \pi^{0} \pi^{0}$ and $\pi^{-} K^{+} \rightarrow \pi^{0} K^{0}$, respectively. Theoretical investigations on the spectrum and the decay of pionium 
have been performed beyond leading order in several settings. In particular, the lifetime of pionium was studied by the use of the Bethe-Salpeter equation ${ }^{7}$ and in

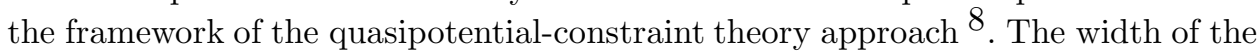
$\pi^{+} \pi^{-}$atom has also been analyzed within a non-relativistic effective field theory 91011, which was originally developed for bound states in general by Caswell and Lepage 12 . The average momenta of the pions circulating in pionium are very small, of the order of $\alpha M_{\pi^{+}}$, where $\alpha$ denotes the fine-structure constant. For this reason, a non-relativistic framework provides the most powerful approach to the calculation of the characteristics of this sort of bound states. In particular, this technique allows one to evaluate the higher order corrections to the Deser-type formulae systematically. In Refs. 910111314 the lifetime of pionium was evaluated at next-to-leading order in the isospin breaking parameters $\alpha \simeq 1 / 137$ and $\left(m_{u}-m_{d}\right)^{2}$. The nonrelativistic framework was further applied to the ground-state of pionic hydrogen $15[1617$ and very recently to the energy-levels and decay widths of kaonic hydrogen 18 .

Within the non-relativistic effective field theory approach we evaluated the Swave decay widths and strong energy shifts of pionium and the $\pi^{ \pm} K^{\mp}$ atom at next-to-leading order in isospin symmetry breaking 1920 . The width of the $\pi^{ \pm} K^{\mp}$ atom is related to the isospin odd $\pi K$ scattering lengths $a_{0}^{-}$, while the strong energy shift is proportional to the sum of isospin even and odd scattering lengths $a_{0}^{+}+a_{0}^{-}$. The values for $a_{0}^{+}$and $a_{0}^{-}$, used in the numerical evaluation of the widths and strong energy shifts, stem from the recent analysis of $\pi K$ scattering from Roy and Steiner type equations 21. Within chiral perturbation theory (ChPT) 22, the $\pi K$ scattering lengths have been worked out at one-loop accuracy 232425), and very recently even the chiral expansion of the $\pi K$ scattering amplitude at next-to-nextto-leading order became available 26 . Particularly interesting is that the isospin even scattering lengths $a_{0}^{+}$depends on the low-energy constant $L_{6}^{r} \frac{22}{27}$, and this coupling is related to the flavour dependence of the quark condensate 27 .

\section{Non-relativistic Lagrangian}

In what follows, we concentrate on the spectrum and decays of the $\pi^{ \pm} K^{\mp}$ atom. The non-relativistic Lagrangian is exclusively determined by symmetries, which are rotational invariance, parity and time reversal. It provides a systematic expansion in powers of the isospin breaking parameter $\delta$. What concerns the $\pi^{-} K^{+}$atom, we count both $\alpha$ and $m_{u}-m_{d}$ as order $\delta$. The different power counting for the $\pi^{+} \pi^{-}$ and $\pi^{-} K^{+}$atoms are due to the fact that in QCD, the chiral expansion of the pion mass difference $\Delta_{\pi}=M_{\pi^{+}}^{2}-M_{\pi^{0}}^{2}$ is of second order in $m_{u}-m_{d}$, while the kaon mass difference $\Delta_{K}=M_{K^{+}}^{2}-M_{K^{0}}^{2}$ starts at first order in $m_{u}-m_{d}$. In the sector with one or two mesons, the non-relativistic $\pi K$ Lagrangian is $\mathcal{L}_{\mathrm{NR}}=\mathcal{L}_{1}+\mathcal{L}_{2}$. The first term contains the one-pion and one-kaon sectors,

$$
\mathcal{L}_{1}=\frac{1}{2}\left(\mathbf{E}^{2}-\mathbf{B}^{2}\right)+h_{0}^{\dagger}\left(i \partial_{t}-M_{h^{0}}+\frac{\Delta}{2 M_{h^{0}}}+\frac{\Delta^{2}}{8 M_{h^{0}}^{3}}+\cdots\right) h_{0}
$$




$$
+\sum_{ \pm} h_{ \pm}^{\dagger}\left(i D_{t}-M_{h^{+}}+\frac{\mathbf{D}^{2}}{2 M_{h^{+}}}+\frac{\mathbf{D}^{4}}{8 M_{h^{+}}^{3}}+\cdots\right) h_{ \pm}
$$

where $\mathbf{E}=-\nabla A_{0}-\dot{\mathbf{A}}, \mathbf{B}=\nabla \times \mathbf{A}$ and the quantity $h=\pi, K$ stands for the non-relativistic pion and kaon fields. We work in the Coulomb gauge and eliminate the $A^{0}$ component of the photon field by the use of the equations of motion. The covariant derivatives are given by $D_{t} h_{ \pm}=\partial_{t} h_{ \pm} \mp i e A_{0} h_{ \pm}$and $\mathbf{D} h_{ \pm}=\nabla h_{ \pm} \pm i e \mathbf{A} h_{ \pm}$, where $e$ denotes the electromagnetic coupling. What concerns the one-pion-one-kaon sector, we only list the terms needed to evaluate the decay width and the energy shift of the $\pi^{-} K^{+}$atom at next-to-leading order in isospin symmetry,

$$
\mathcal{L}_{2}=C_{1}^{\prime} \pi_{-}^{\dagger} K_{+}^{\dagger} \pi_{-} K_{+}+C_{2}\left(\pi_{-}^{\dagger} K_{+}^{\dagger} \pi_{0} K_{0}+\text { h.c }\right)+C_{3} \pi_{0}^{\dagger} K_{0}^{\dagger} \pi_{0} K_{0}+\cdots
$$

The coupling constant $C_{1}^{\prime}$ contains contributions coming from the electromagnetic form factors of the pion and kaon,

$$
C_{1}^{\prime}=C_{1}-e^{2} \lambda, \quad \lambda=\frac{1}{6}\left(\left\langle r_{\pi^{+}}^{2}\right\rangle+\left\langle r_{K^{+}}^{2}\right\rangle\right),
$$

where $\left\langle r_{\pi^{+}}^{2}\right\rangle$ and $\left\langle r_{K^{+}}^{2}\right\rangle$ denote the charge radii of the charged pion and kaon, respectively. The low energy constants $C_{1}, \ldots, C_{3}$ are determined through matching to the relativistic theory. For illustration we give the result of the matching in the isospin symmetry limit:

$$
C_{1}=\frac{2 \pi}{\mu_{+}}\left(a_{0}^{+}+a_{0}^{-}\right), \quad C_{2}=-\frac{2 \sqrt{2} \pi}{\mu_{+}} a_{0}^{-}, \quad C_{3}=\frac{2 \pi}{\mu_{+}} a_{0}^{+},
$$

where the S-wave scattering lengths ${ }^{\mathrm{a}} a_{0}^{+}=1 / 3\left(a_{0}^{1 / 2}+2 a_{0}^{3 / 2}\right)$ and $a_{0}^{-}=1 / 3\left(a_{0}^{1 / 2}-\right.$ $\left.a_{0}^{3 / 2}\right)$ are defined in $\mathrm{QCD}$, at $m_{u}=m_{d}$ and $M_{\pi} \doteq M_{\pi^{+}}, M_{K} \doteq M_{K^{+}}$.

\section{Decay width of the $\pi K$ atom}

To evaluate the energy shift and decay width of the $\pi^{-} K^{+}$atom at next-to-leading order in isospin symmetry breaking, we make use of resolvents. For a detailed discussion of the technique, we refer to Ref. 20 . Here, we simply list the results. The decay width of the ground-state reads at order $\delta^{9 / 2}$, in terms of the relativistic $\pi^{-} K^{+} \rightarrow \pi^{0} K^{0}$ threshold amplitude 1920 ,

$$
\Gamma=8 \alpha^{3} \mu_{+}^{2} p^{*} \mathcal{A}^{2}(1+K), \quad \mathcal{A}=-\frac{1}{8 \sqrt{2} \pi} \frac{1}{M_{\pi^{+}}+M_{K^{+}}} \operatorname{Re} A_{\mathrm{thr}}^{00 ; \pm}+o(\delta),
$$

where $\mu_{+}$denotes the reduced mass of the $\pi K$ atom and

$$
K=\frac{M_{\pi^{+}} \Delta_{K}+M_{K^{+}} \Delta_{\pi}}{M_{\pi^{+}}+M_{K^{+}}}\left(a_{0}^{+}\right)^{2}-4 \alpha \mu_{+}\left(a_{0}^{+}+a_{0}^{-}\right)[\ln \alpha-1]+o(\delta) .
$$

The outgoing relative 3 -momentum

$$
p^{*}=\frac{1}{2 E_{1}} \lambda\left(E_{1}^{2}, M_{\pi^{0}}^{2}, M_{K^{0}}^{2}\right)^{1 / 2},
$$

${ }^{\mathrm{a}} a_{0}^{+}$and $a_{0}^{-}$are normalized as in Ref. 23 . 
with $\lambda(x, y, z)=x^{2}+y^{2}+z^{2}-2 x y-2 x z-2 y z$, is chosen such that the total final state energy corresponds to ground-state Coulomb energy $E_{1}=M_{\pi^{+}}+M_{K^{+}}-\alpha^{2} \mu_{+} / 2$. The quantity $\operatorname{Re} A_{\mathrm{thr}}^{00 ; \pm}$ is calculated as follows. One evaluates the relativistic $\pi^{-} K^{+} \rightarrow \pi^{0} K^{0}$ amplitude at order $\delta$ near threshold and removes the divergent Coulomb phase. The real part contains singularities $\sim 1 /|\mathbf{p}|$ and $\sim \ln |\mathbf{p}| / \mu_{+}$. The constant term in this expansion corresponds to $\operatorname{Re} A_{\mathrm{thr}}^{00 ; \pm}$. The normalization is chosen such that

$$
\mathcal{A}=a_{0}^{-}+\epsilon .
$$

The isospin breaking corrections $\epsilon$ are calculable within ChPT and have been evalu-

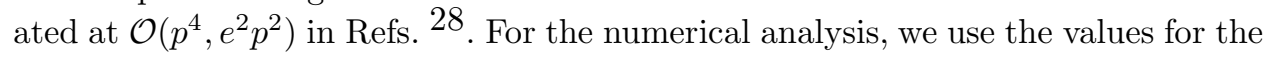
$\pi K$ scattering lengths from the recent analysis of data and Roy-Steiner equations 21] $a_{0}^{+}=(0.045 \pm 0.012) M_{\pi^{+}}^{-1}$ and $a_{0}^{-}=(0.090 \pm 0.005) M_{\pi^{+}}^{-1}$. At next-to-leading order in isospin symmetry breaking the prediction for the lifetime of the $\pi^{ \pm} K^{\mp}$ atom in its ground-state amounts to 20

$$
\tau \doteq \Gamma^{-1}=(3.7 \pm 0.4) \cdot 10^{-15} \mathrm{~s} .
$$

The bulk part in the uncertainty is due to the uncertainties in the $\pi K$ scattering lengths.

\section{Energy splittings}

Table 1. Numerical values for the energy shift of the $\pi^{ \pm} K^{\mp}$ atom.

\begin{tabular}{cccc}
\hline$\pi^{ \pm} K^{\mp}$ atom & $\Delta E_{n l}^{\mathrm{em}}[\mathrm{eV}]$ & $\Delta E_{n l}^{\mathrm{vac}}[\mathrm{eV}]$ & $\Delta E_{n l}^{\mathrm{h}}[\mathrm{eV}]$ \\
\hline$n=1, l=0$ & -0.095 & -2.56 & $-9.0 \pm 1.1$ \\
$n=2, l=0$ & -0.019 & -0.29 & $-1.1 \pm 0.1$ \\
$n=2, l=1$ & -0.006 & -0.02 & \\
\hline
\end{tabular}

The energy-level splittings of the $\pi^{ \pm} K^{\mp}$ atom are induced by both electromagnetic and strong interactions. At order $\delta^{3}$, the energy shift contributions are exclusively due to strong interactions, while at order $\delta^{4}$, both electromagnetic and strong interactions contribute. It is both conventional and convenient to split the energy shifts into a strong and an electromagnetic part, according to ${ }^{\mathrm{b}}$

$$
\Delta E_{n l}=\Delta E_{n l}^{\mathrm{h}}+\Delta E_{n l}^{\mathrm{em}},
$$

where $n$ stands for the principal quantum number and $l$ for the angular momentum. The electromagnetic energy shift contains both pure QED corrections as well as finite size effects due to the charge radii of the pion and kaon, see Eq. (3). The pure

${ }^{b}$ Note that this splitting cannot be understood literally, i.e. there are contributions from strong interactions to $\Delta E_{n 0}^{\mathrm{em}}$. 
electromagnetic corrections have been evaluated a long time ago by Nandy 29 and the finite size effects are given in 20. For the first two states the numerical values for $\Delta E_{n l}^{\mathrm{em}}$ are listed in Table 1

At order $\delta^{4}$, the strong S-wave energy shift yields1920,

$$
\Delta E_{n 0}^{\mathrm{h}}=-\frac{2 \alpha^{3} \mu_{+}^{2}}{n^{3}} \mathcal{A}^{\prime}\left(1+K_{n}^{\prime}\right), \quad \mathcal{A}^{\prime}=\frac{1}{8 \pi\left(M_{\pi^{+}}+M_{K^{+}}\right)} \operatorname{Re} A_{\mathrm{thr}}^{ \pm ; \pm}+o(\delta),
$$

where

$$
K_{n}^{\prime}=-2 \alpha \mu_{+}\left(a_{0}^{+}+a_{0}^{-}\right)\left[\psi(n)-\psi(1)-\frac{1}{n}+\ln \frac{\alpha}{n}\right]+o(\delta),
$$

and $\psi(n)=\Gamma^{\prime}(n) / \Gamma(n)$. The quantity $\operatorname{Re} A_{\mathrm{thr}}^{ \pm ; \pm}$is determined by the constant term occurring in the threshold expansion of the relativistic $\pi^{-} K^{+} \rightarrow \pi^{-} K^{+}$amplitude. In the isospin limit, the normalized relativistic amplitude

$$
\mathcal{A}^{\prime}=a_{0}^{+}+a_{0}^{-}+\epsilon^{\prime}
$$

reduces to the sum of the isospin even and odd scattering lengths. The corrections $\epsilon^{\prime}$ have been evaluated within $\mathrm{ChPT}$ at $\mathcal{O}\left(p^{4}, e^{2} p^{2}\right)$ in Refs. 25 . The result for $\Delta E_{10}^{\mathrm{h}}$ in Eq. (11) agrees with the one obtained for the strong energy shift of the ground-state in pionic hydrogen 15, if we replace $\mu_{+}$with the reduced mass of the $\pi^{-} p$ atom and $\operatorname{Re} A_{\mathrm{thr}}^{ \pm ;}$with the constant term in the threshold expansion for the real part of the truncated $\pi^{-} p \rightarrow \pi^{-} p$ amplitude. The numerical values for the energy splittings of the first two states are listed in Table 1 where again the values for $a_{0}^{+}$and $a_{0}^{-}$are taken from Ref. 21 .

What remains to be added are the contributions coming from the electron vacuum polarization. The calculation of these corrections within a non-relativistic Lagrangian approach has been performed in Ref. 10. In our framework, the contributions due to vacuum polarization arise formally at higher order in $\alpha$. However, they are amplified by powers of the coefficient $\mu_{+} / m_{e}$, where $m_{e}$ denotes the electron mass. For the first two energy-level shifts, the vacuum polarization contributions $\Delta E_{n l}^{\mathrm{vac}}$ are listed in Table 1

\section{Summary}

We provided the formulae for the energy shift and the decay width of the $\pi^{ \pm} K^{\mp}$ atom at next-to-leading order in isospin symmetry breaking. At leading and next-to leading order the $\pi^{-} K^{+}$atom decays into $\pi^{0} K^{0}$ exclusively. Aside from a kinematical factor - the relativistic outgoing 3-momentum of the bound system - the width can be expanded in powers of $\alpha$ and $m_{u}-m_{d}$. The decay width is related to $\pi^{-} K^{+} \rightarrow \pi^{0} K^{0}$ threshold amplitude, which is determined in the isospin limit by the isospin odd scattering length $a_{0}^{-}$. The isospin breaking contributions to this amplitude have been evaluated at $\mathcal{O}\left(e^{2} p^{2}, p^{4}\right)^{28}$ in the framework of ChPT. By invoking ChPT, the result for the decay width of the $\pi^{-} K^{+}$atom in its groundstate may be expressed in terms of the isospin odd scattering length $a_{0}^{-}$, and isospin symmetry breaking corrections of order $\alpha$ and $m_{u}-m_{d}$, see Eq. (15). 
It is both conventional and convenient to split the energy shifts into an electromagnetic and a strong part, according to Eq. (10). The electromagnetic part contains both pure QED contributions as well as finite size effects due to the charge radii of the pion and kaon. The strong energy shift of the $\pi^{-} K^{+}$atom is proportional to the one-particle irreducible $\pi^{-} K^{+} \rightarrow \pi^{-} K^{+}$scattering amplitude at threshold. In the isospin symmetry limit, the elastic threshold amplitude reduces to the sum of isospin even and odd scattering lengths $a_{0}^{+}+a_{0}^{-}$. The isospin breaking contributions to the amplitude have been evaluated at $\mathcal{O}\left(e^{2} p^{2}, p^{4}\right)^{25}$ in the framework of ChPT. The result in Eq. (11) displays the S-wave energy shift in terms of the sum $a_{0}^{+}+a_{0}^{-}$, and corrections of order $\alpha$ and $m_{u}-m_{d}$.

Our prediction for the lifetime of the $\pi^{ \pm} K^{\mp}$ atom in its ground-state yields: $\tau=(3.7 \pm 0.4) \cdot 10^{-15} \mathrm{~s}$, while the numerical values for the energy shifts are listed in Table 1 To confront these predictions with data presents a challenge for future hadronic atom experiments.

\section{Acknowledgments}

It is a pleasure to thank J. Gasser, A. Rusetsky, H. Sazdjian and J. Schacher for many helpful discussions. This work was supported in part by the Swiss National Science Foundation and by RTN, BBW-Contract N0. 01.0357 and EC-Contract HPRN-CT2002-00311 (EURIDICE).

\section{References}

1. B. Adeva et al., CERN-SPSLC-95-1; see also D. Goldin, these proceedings.

2. PSI experiment R-98.01, http://pihydrogen.web.psi.ch see also D. Gotta, these proceedings.

3. S. Bianco et al. [DEAR Collaboration], Riv. Nuovo Cim. 22N11 (1999) 1; see also M. Cargnelli, these proceedings.

4. S. Deser, M. L. Goldberger, K. Baumann and W. Thirring, Phys. Rev. 96 (1954) 774.

5. T. R. Palfrey and J. L. Uretsky, Phys. Rev. 121 (1961) 1798.

6. S. M. Bilenky, V. H. Nguyen, L. L. Nemenov and F. G. Tkebuchava, Yad. Fiz. 10 (1969) 812 [Sov. J. Nucl. Phys. 10 (1969) 469].

7. V. E. Lyubovitskij and A. Rusetsky, Phys. Lett. B 389 (1996) 181, arXiv:hep-ph/9610217; V. E. Lyubovitskij, E. Z. Lipartia and A. G. Rusetsky, Sov. Phys. JETP Lett. 66 (1997) 783, arXiv:hep-ph/9801215; M. A. Ivanov, V. E. Lyubovitskij, E. Z. Lipartia and A. G. Rusetsky, Phys. Rev. D 58 (1998) 094024, arXiv:hep-ph/9805356.

8. H. Jallouli and H. Sazdjian, Phys. Rev. D 58 (1998) 014011 [Erratum-ibid. D 58 (1998) 099901], arXiv:hep-ph/9706450; H. Sazdjian, arXiv:hep-ph/9809425; Phys. Lett. B 490 (2000) 203, arXiv:hep-ph/0004226.

9. P. Labelle and K. Buckley, arXiv:hep-ph/9804201; X. Kong and F. Ravndal, Phys. Rev. D 59 (1999) 014031; Phys. Rev. D 61 (2000) 077506, arXiv:hep-ph/9905539; B. R. Holstein, Phys. Rev. D 60 (1999) 114030, arXiv:nucl-th/9901041; A. Gall, J. Gasser, V. E. Lyubovitskij and A. Rusetsky, Phys. Lett. B 462 (1999) 335, arXiv:hep-ph/9905309 ; D. Eiras and J. Soto, Phys. Rev. D 61 (2000) 114027, arXiv:hep-ph/9905543 ; V. Antonelli, A. Gall, J. Gasser and A. Rusetsky, Ann. Phys. (NY) 286 (2001) 108, arXiv:hep-ph/0003118. 
10. D. Eiras and J. Soto, Phys. Lett. B 491 (2000) 101, arXiv:hep-ph/0005066.

11. J. Gasser, V. E. Lyubovitskij, A. Rusetsky and A. Gall, Phys. Rev. D 64 (2001) 016008, arXiv:hep-ph/0103157.

12. W. E. Caswell and G. P. Lepage, Phys. Lett. B 167 (1986) 437.

13. H. W. Hammer and J. N. Ng, Eur. Phys. J. A 6 (1999) 115, arXiv:hep-ph/9902284.

14. J. Gasser, V. E. Lyubovitskij and A. Rusetsky, Phys. Lett. B 471 (1999) 244, arXiv:hep-ph/9910438.

15. V. E. Lyubovitskij and A. Rusetsky, Phys. Lett. B 494 (2000) 9, arXiv:hep-ph/0009206.

16. J. Gasser, M. A. Ivanov, E. Lipartia, M. Mojzis and A. Rusetsky, Eur. Phys. J. C 26 (2002) 13, arXiv:hep-ph/0206068.

17. P. Zemp, in: Proceedings of the Workshop HadAtom03, 13-17 October 2003, ETC* (Trento, Italy), J. Gasser, A. Rusetsky and J. Schacher, Eds., p. 18, arXiv:hep-ph/0401204.

18. U. G. Meissner, U. Raha and A. Rusetsky, arXiv:hep-ph/0402261.

19. J. Schweizer, Phys. Lett. B 587 (2004) 33 arXiv:hep-ph/0401048.

20. J. Schweizer, arXiv:hep-ph/0405034

21. P. Buettiker, S. Descotes-Genon and B. Moussallam, Eur. Phys. J. C 33 (2004) 409 arXiv:hep-ph/0310283.

22. J. Gasser and H. Leutwyler, Nucl. Phys. B 250 (1985) 465.

23. V. Bernard, N. Kaiser and U. G. Meissner, Phys. Rev. D 43 (1991) 2757.

24. A. Roessl, Nucl. Phys. B 555 (1999) 507, arXiv:hep-ph/9904230.

25. A. Nehme, Eur. Phys. J. C 23 (2002) 707, arXiv:hep-ph/0111212; B. Kubis and U. G. Meissner, Phys. Lett. B 529 (2002) 69, arXiv:hep-ph/0112154.

26. J. Bijnens, P. Dhonte and P. Talavera, arXiv:hep-ph/0404150 .

27. S. Descotes-Genon, L. Girlanda and J. Stern, J. High Energy Phys. 0001 (2000) 041, arXiv:hep-ph/9910537; B. Moussallam, J. High Energy Phys. 0008 (2000) 005, arXiv:hep-ph/0005245 ; S. Descotes-Genon, L. Girlanda and J. Stern, Eur. Phys. J. C 27 (2003) 115, arXiv:hep-ph/0207337; S. Descotes-Genon, N. H. Fuchs, L. Girlanda and J. Stern, arXiv:hep-ph/0311120.

28. B. Kubis and U. G. Meissner, Nucl. Phys. A 699 (2002) 709 arXiv:hep-ph/0107199;

A. Nehme and P. Talavera, Phys. Rev. D 65 (2002) 054023 arXiv:hep-ph/0107299.

29. A. Nandy, Phys. Rev. D 5 (1972) 1531. 